NIST Advanced Manufacturing Series 100-27

\title{
Influence of Optical System Operation on Stability of Single Tracks in Selective Laser Melting
}

\author{
Ivan Zhirnov \\ Igor Yadroitsev \\ Brandon Lane \\ Sergey Mekhontsev \\ Steven Grantham \\ Ina Yadroitsava
}

This publication is available free of charge from:

https://doi.org/10.6028/NIST.AMS.100-27

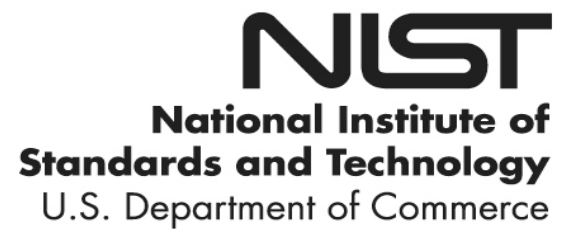




\title{
Influence of Optical System Operation on Stability of Single Tracks in Selective Laser Melting
}

\author{
Ivan Zhirnov \\ Sergey Mekhontsev \\ Steven Grantham \\ Sensor Science Division \\ Physical Measurement Laboratory \\ Igor Yadroitsev \\ Ina Yadroitsava \\ Department of Mechanical and Mechatronics Engineering \\ Central University of Technology, Free State, South Africa \\ Brandon Lane \\ Intelligent Systems Division \\ Engineering Laboratory
}

This publication is available free of charge from:

https://doi.org/10.6028/NIST.AMS.100-27

August 2019

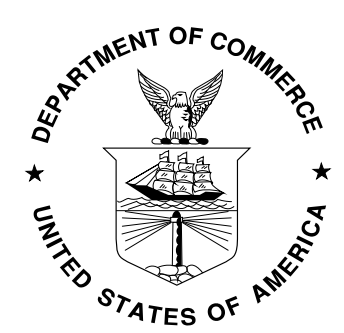

U.S. Department of Commerce Wilbur L. Ross, Jr., Secretary

National Institute of Standards and Technology Walter Copan, NIST Director and Undersecretary of Commerce for Standards and Technology 
Certain commercial entities, equipment, or materials may be identified in this document in order to describe an experimental procedure or concept adequately. Such identification is not intended to imply recommendation or endorsement by the National Institute of Standards and Technology, nor is it intended to imply that the entities, materials, or equipment are necessarily the best available for the purpose.

National Institute of Standards and Technology Advanced Manufacturing Series 100-27

Natl. Inst. Stand. Technol. Adv. Man. Ser. 100-27, 14 pages (August 2019)

This publication is available free of charge from: https://doi.org/10.6028/NIST.AMS.100-27 


\begin{abstract}
Additive manufacturing (AM) technologies are increasingly being studied and introduced into the modern industry, but for wide applications there exists some "lack of confidence" about the quality of the parts produced by AM. This distrust has an objective basis: it was shown that the final 3D object is a superposition of a huge number of tracks and layers, and deviations from the optimal process parameters can lead to non-regular shape, unmelted places (lack of fusion) and porosity. Experiments with different bare substrates were performed to classify instabilities and artifacts in single tracks derived from laser beam characteristics, the optical system, scanning strategy, etc. demonstrating an adjustment scheme for testing and verifying LPBF equipment. An important point is that this research can be useful for the custom experimental setup. The paper describes the importance of checking the system before each build to identify problems caused by optical system operation. Also, possible deviations from the stable process, methods of their diagnostics and solutions are described. The proposed method is a cost-free way to diagnose the stability of the selective laser melting, which does not imply the necessity of having additional systems for detecting problems. The diagnostic scheme was used to evaluate in situ diagnostics of the LPBF processes on the Additive Manufacturing Metrology Testbed (AMMT) at the National Institute of Standards and Technology (NIST).
\end{abstract}

\title{
Key words
}

metal additive manufacturing; selective laser melting; quality control; diagnostics. 


\section{Table of Contents}

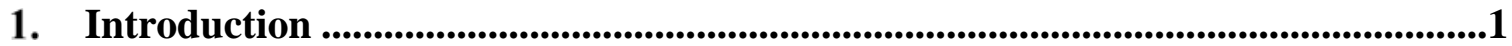

2. Experimental setup...........................................................................................................1

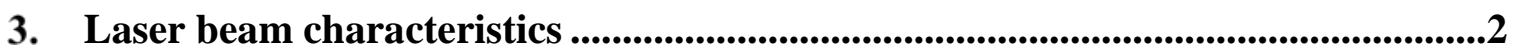

4. Thermal effects and contaminations of optical components .....................................4

5. Verification of galvanometer scanner...............................................................5

6. Test patterns for verification of scanning system, laser beam, and optical components .......................................................................................................................7

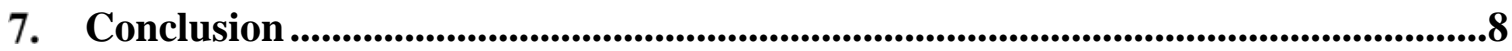

References....................................................................................................................................8

\section{List of Tables}

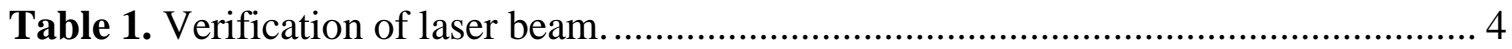

Table 2. Verification of optical system. ……………….............................................. 6

Table 3. Verification of scanner........................................................................... 7

\section{List of Figures}

Fig. 1. Optical scheme of experimental set-up at NIST.

Fig. 2. The instability of laser radiation influences on the laser track formation. Similar laser power and scanning speed lead to different results when Ti6Al4V substrate was scanned with Gaussian single-mode laser beam (a) and in case of incorrect laser operation (b). Samples manufactured with EOSINT M270.

Fig. 3. Two single tracks on a platinum substrate manufactured at the same prescribed laser power and scanning speed. For the irregular track, the laser-optical system did not work correctly: (a) laser power drop and (b) contamination of optical system and thermal effect. The sample was manufactured using a commercial EOS M280. 3 Fig. 4. Single tracks on a bare substrate manufactured at (a, b, c) $100 \mu \mathrm{m}$ spot size and $200 \mathrm{~W}$ laser power and (d, e, f) $170 \mu \mathrm{m}$ spot size and $578 \mathrm{~W}$ laser power. Samples manufactured using experimental set-up by NIST.

Fig. 5. Non-straight stainless-steel single track on the substrate. Samples manufactured using experimental testbed set-up by NIST.

Fig. 6. Proposed test plate designs for verification of scanning system, laser beam, and optical components. Direction of scanning indicated by arrows, black lines are scanning at lower scanning speed and laser power, red lines are scanning at higher scanning speed and laser power. 


\section{Introduction}

Laser powder bed fusion (LPBF), or selective laser melting (SLM), provides unchallenged freedom of design that allows complex parts to be created in one production cycle which are nearly impossible to manufacture using conventional processes such as turning, milling, or casting. The high degree of freedom offered by LPBF technology enables production of objects having complex geometries and internal structure such as cooling channels or sophisticated lattice structures. Selective energy input, high-temperature gradients, and high cooling rates produce materials with unique properties. This reveals new opportunities in the automotive, energy, aerospace, medical, tooling, and other advanced industries.

To produce high-quality non-porous objects with reliable mechanical properties and dimensional accuracy using this technology, optimal process parameters and conditions for powder materials are required. During processing, all units such as laser beam characteristics, scanner, protective windows, and splitters (i.e., all optical components) can have an influence on the results. Experiments that were done in the Engineering Laboratory at the National Institute of Standards and Technology (USA) and in the Central University of Technology (Free State, South Africa) were used to identify single track instabilities and their causes.

\section{Experimental setup}

NIST developed an open platform LPBF testbed called the Additive Manufacturing Metrology Testbed (AMMT) and Temperature and Emittance of Melts, Powders and Solids (TEMPS) facilities. This complex system allows monitoring and control of system performance, properties of bulk and powder materials, melt pool, sintered tracks and surfaces, etc. Optical design of the experimental setup for LPBF-type measurements is shown in Fig. 1. The optical scanner, build area, powder management, and sample motion are contained within a $1750 \mathrm{~mm} \times 1200 \mathrm{~mm} \times 780 \mathrm{~mm}$ sealed process chamber. The scanning area is $100 \mathrm{~mm} \times 100 \mathrm{~mm}$, and the processed chamber is evacuated and back-filled with inert gas. All optical transmission elements are antireflection coated. The lenses are made of high transmittance (HT) type Schott glasses ${ }^{1}$ which have reduced absorption and scatter loss compared to their non-HT offerings. The Broadband Dielectric Mirror has a coating $750 \mathrm{~nm}$ to $1100 \mathrm{~nm}$ on fused silica substrates. The laser mirror has greater than $99.98 \%$ reflectivity at $1064 \mathrm{~nm}$ wavelength on ultraviolet fused silica substrates. The fused silica beam splitter has $90 \%$ transmission at $532 \mathrm{~nm}$ wavelength and 99.5\% reflection at $1064 \mathrm{~nm}$ wavelength. Fused silica (FS) is used for protective windows. A detailed description of the NIST platform testbed can be found in Grantham et al. (2016) [1].

\footnotetext{
${ }^{1}$ Certain commercial equipment, instruments, or materials are identified in this paper to foster understanding. Such identification does not imply recommendation or endorsement by the National Institute of Standards and Technology, nor does it imply that the materials or equipment identified are necessarily the best available for the purpose.
} 


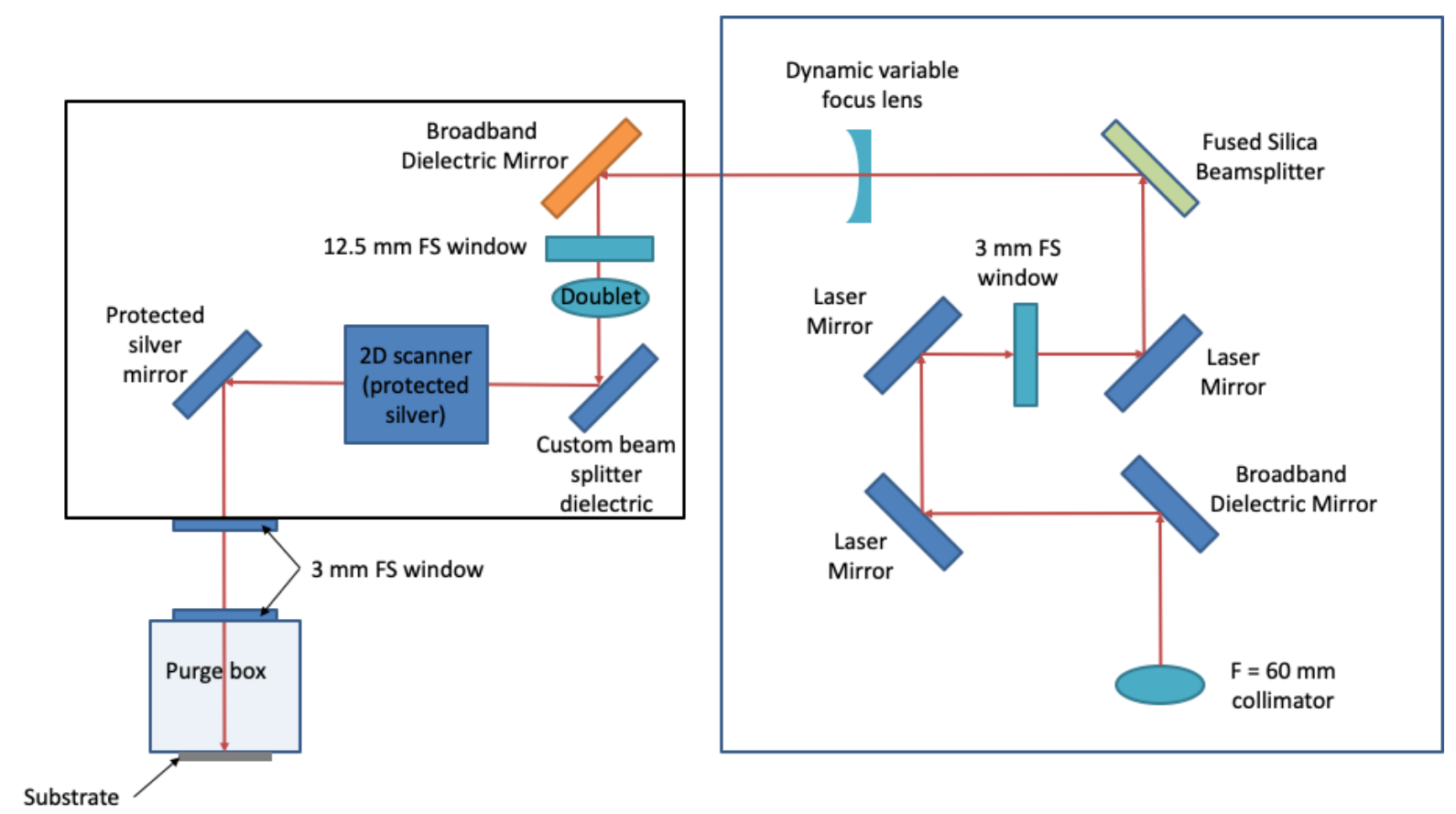

Fig. 1. Optical scheme of experimental set-up at NIST.

In typical commercial powder bed fusion systems, the optical system that creates and positions the laser beam to sinter powder material also consists of an Ytterbium (Yb) fiber laser with wavelength $1060 \mathrm{~nm}$ to $1100 \mathrm{~nm}$, beam expander optic, scanner mirrors, and F-theta objective lens. All optical surfaces have special coatings. The high-speed scanner (up to $7 \mathrm{~m} / \mathrm{s}$ scanning speed) guarantees precise and stable work at the building area of $250 \mathrm{~mm} \times 250 \mathrm{~mm}$. The optical system includes an F-theta objective and protective glass with a pneumatic lens protection device which prevents dirt from settling on the lens surface [2].

\section{Laser beam characteristics}

The laser beam and optical system are crucial for LPBF because they define the shape, penetration depth, and stability of each scan track. Stability is particularly important as shown in Fig. 2.

The beam quality must be specified at the initial step of any laser processing accordingly to ISO 13694 (2000) [3] and ISO 11146 (1999) [4] standards: focus diameter, focus position, divergence, ellipticity, beam propagation factor, etc. [5]. Zhirnov et al. showed that using different shapes of laser beam, i.e., different power density distributions, single tracks exhibit differences both in microstructure and track geometry [6]. Beam quality, power density, and intensity profile all influence LPBF processing quality. Yadroitsev et al., Makoana et al., and Shi et al. showed that in LPBF, the width of the tracks is primarily defined by spot size and laser power [7-9]. During laser processing, the diameter of the laser beam defines the power density and, as a result, geometrical characteristics of single tracks. 


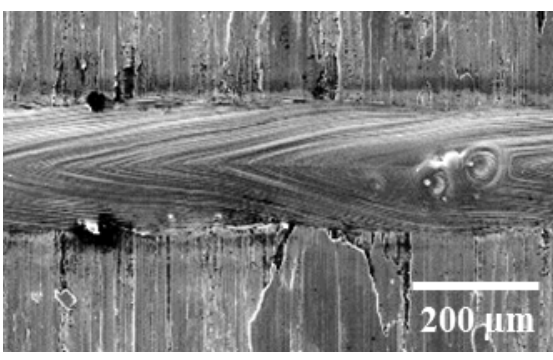

(a)

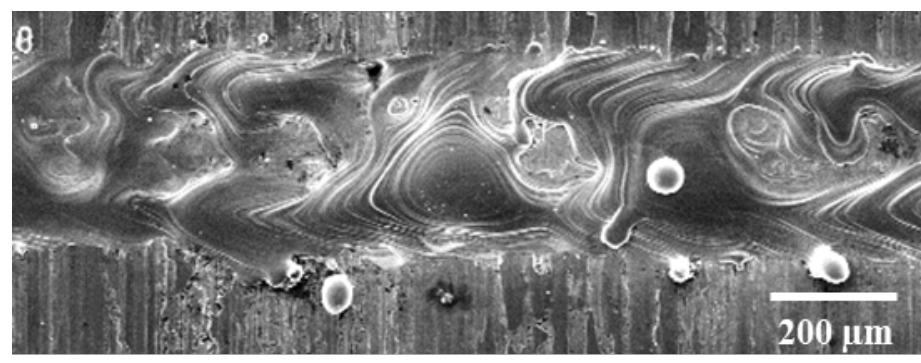

(b)

Fig. 2. The instability of laser radiation influences on the laser track formation. Similar laser power and scanning speed lead to different results when Ti6Al4V substrate was scanned with Gaussian single-mode laser beam (a) and in case of incorrect laser operation (b). Samples manufactured with EOSINT M270.

In the optical system, dynamic laser heating and contamination influences the stability of the LPBF process, so properties of the optical components and protective window are important [10]. When the laser operates in unstable transverse electromagnetic mode (TEM) or the optical system absorbs laser beam energy, laser power delivered by the optical system will not be uniform and constant to form stable tracks, and the same process parameters will result in track-to-track differences (Fig. 3).

Similar artifacts to those shown in Fig. 3 were found on a 17-4 stainless steel substrate by scanning the laser beam with varying spot sizes while maintaining laser power density (Fig. 4). This may be due to a non-Gaussian laser beam profile and changing of the mode of generating when higher-order modes are generated with complex intensity profiles. For example, for the cases shown in Fig. 4 (b, e), it looks like transverse electromagnetic TEM $\mathrm{T}_{01}$ laser mode with two adjacent spots generated the tracks.

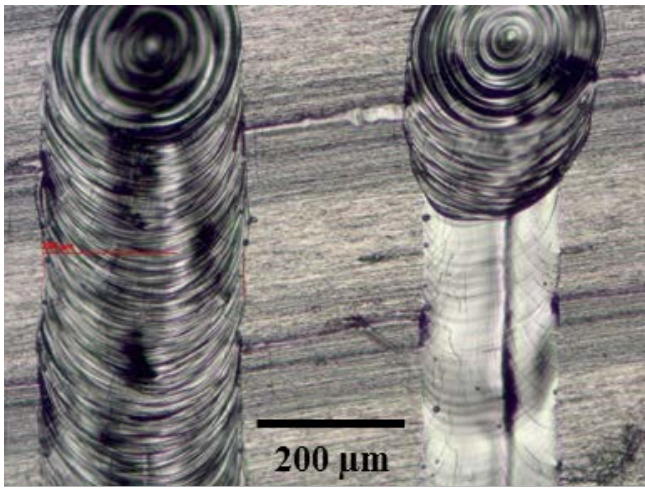

(a)

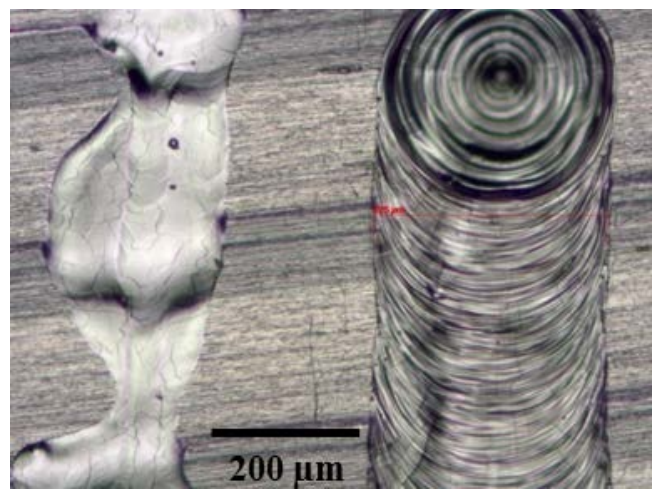

(b)

Fig. 3. Two single tracks on a platinum substrate manufactured at the same prescribed laser power and scanning speed. For the irregular track, the laser-optical system did not work correctly: (a) laser power drop and (b) contamination of optical system and thermal effect. The sample was manufactured using a commercial EOS M280.

Unstable generation of the laser beam resulted in further differences between track shapes produced with similar prescribed laser power, spot size, and scanning speeds 
(Fig. 4c, f). To diagnose these issues, different approaches can be used. First, full verification of the system and laser scanning of the substrate must be performed (Table 1). At high thermal loads on the laser fiber, thermal lensing effects change the effective mode area significantly and leads to higher peak intensity. When thermal effects are large enough, it can induce multi-mode behavior and change a fiber from the single-mode TEM 00 regime to a multimode regime [11].

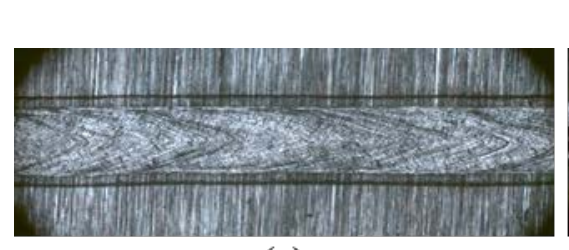

(a)

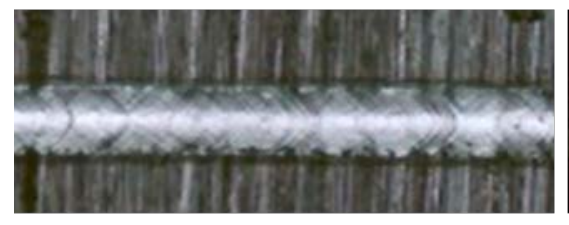

(d)

\section{$\leftarrow$ Scanning direction}

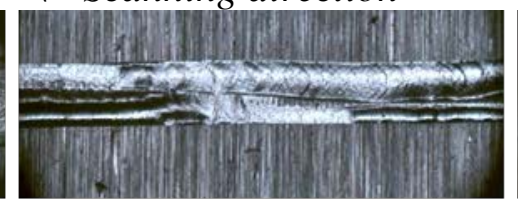

(b)

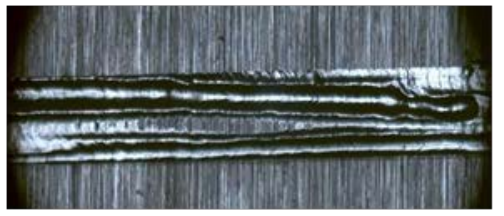

(e)

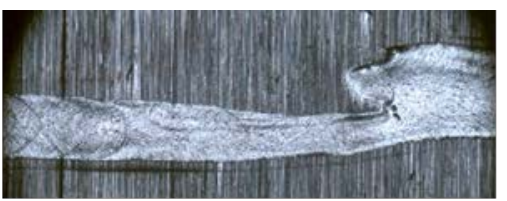

(c)

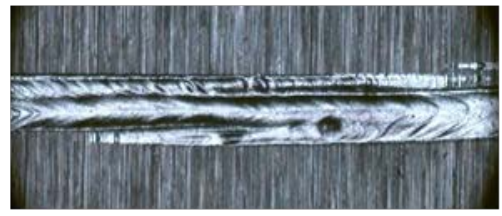

(f)

$\overline{200 \mu \mathrm{m}}$

Fig. 4. Single tracks on a bare substrate manufactured at (a, b, c) $100 \mu \mathrm{m}$ spot size and $200 \mathrm{~W}$ laser power and (d, e, f) $170 \mu \mathrm{m}$ spot size and $578 \mathrm{~W}$ laser power. Samples manufactured using experimental set-up by NIST.

Table 1. Verification of laser beam.

Step 1. Check intensity profile of collimated laser beam without additional optical system

TEM 00 mode?

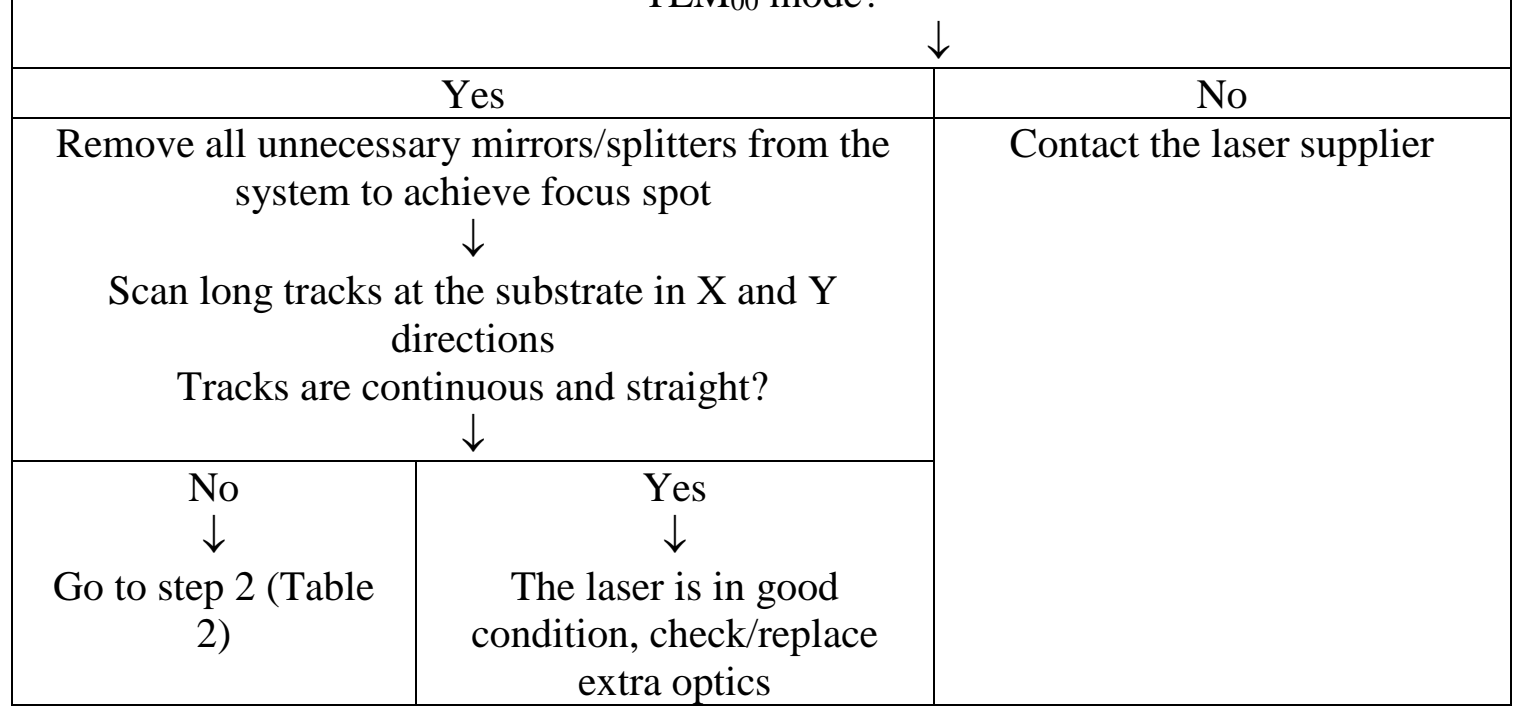

\section{Thermal effects and contaminations of optical components}

Irregular behavior of the sintered tracks suggests that focus shift and aberrations changed the spot size, and re-distributed laser energy intensity. During laser processing, 
thermal effects and contaminations of optical components result in variable spot size and intensity distribution.

Thermal effects from the protective windows were shown by Faidel et al. [10]. It was found that at $25 \mathrm{~W}$ laser power (no thermal effects) and $250 \mathrm{~W}$ (with thermal effects), material for protective windows affected the laser power profile. Windows from athermalized glass demonstrate reduction of negative thermal effects because laser beam waist (focal depth) shift was significantly lower, and intensity distributions and spot sizes were practically constant in all working planes in contrast to protective windows from borosilicate glass (Shott). This statement was supported by numerical simulations and experimental work by Bonhoff et al. [12]. They showed an increasing contamination of the protective window made from fused silica was accompanied by an increasing focus shift and a decreasing beam quality. As indicated by Faidel et al., fused silica can be used for collimating and focusing optics due to low absorption, but in ordinary plane-surface protective windows, contamination has strong thermal effects [10]. It was recommended to use self-compensating windows from special glasses (athermalized protection windows) to provide smaller thermal waist shift and diffraction limited focusing.

\section{Verification of galvanometer scanner}

As it was shown by Yeung et al., laser scanning path, laser power, and scanning speed have to be strictly controlled during laser processing [13]. Choosing different scanning strategy influences the shape of sintered tracks, single layers, and the whole three-dimensional sample. All else being equal, the correct operation of the scanner is primarily important (Fig. 5).

A scanner vendor indicated in their guidance on problems with the dynAXIS galvanometer scanner that damaged or dirty mirrors are the main causes of low laser power or changed laser spot [14]. Therefore, step 2 in the LPBF system diagnosis must be verification of the system to produce long stable tracks in different directions (Table 2).

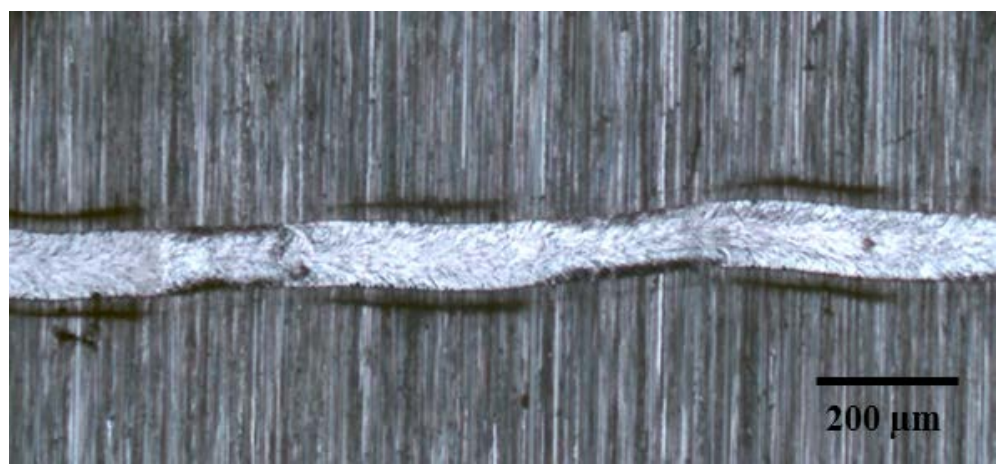

Fig. 5. Non-straight stainless-steel single track on the substrate. Samples manufactured using experimental testbed set-up by NIST. 
Table 2. Verification of optical system.

\begin{tabular}{|c|c|}
\hline \multicolumn{2}{|c|}{$\begin{array}{l}\text { Step 2. Use all optical elements. Scan long tracks at the substrate in } \mathrm{X} \text { and } \\
\text { at the laser power and spot size that was used for first step. } \\
\text { Analyze shape of the tracks and compare with previous test. } \\
\text { Tracks are continuous? } \\
\downarrow\end{array}$} \\
\hline Yes & $\begin{array}{c}\text { No } \\
\downarrow\end{array}$ \\
\hline \multirow[t]{2}{*}{$\downarrow$} & $\begin{array}{c}\text { Check protection windows for the } \\
\text { existence of damage or dirt } \\
\downarrow\end{array}$ \\
\hline & $\begin{array}{c}\text { Check gas flow manner to decrease } \\
\text { contamination for the oxygen level in } \\
\text { work space } \\
\downarrow\end{array}$ \\
\hline \multicolumn{2}{|c|}{ Tracks are straight? } \\
\hline $\begin{array}{c}\downarrow \\
\text { Yes }\end{array}$ & $\begin{array}{c}\downarrow \\
\text { No }\end{array}$ \\
\hline & Go to Step 3 (Table 3) \\
\hline
\end{tabular}

The precise synchronization of mirror motion and the laser switching times is one of the major factors that influence LPBF. An example of synchronization calibration was proposed by Koglbauer et al., who proposed a novel device that uses a window with an etched pattern with a precise geometry on a diffuser [15]. Two photodiodes measured laser light scattered from the pattern or diffuser, and precise time-resolved signals were related to transient changes in scan speed or laser power within a scan vector.

Oscillation or noise while scanning can occur from mechanical oscillations of the mirror perpendicular or parallel to the scan direction [16]. In other cases, when the motors of the mirrors (galvanometers) are working from one power supply, cross-talk between them or when peak drive current capability of power supply is low can result in slight movement in one axis. Also, sometimes the cause of residual oscillations of the mirrors can be a sharp change in the scanning speed by a perturbation in the analog or digital galvometer command signal. Thus, stability of the scanner is very important for single track processing (Table 3). 
Table 3. Verification of scanner.

\begin{tabular}{|c|c|}
\hline \multicolumn{2}{|c|}{ Software configured correctly? } \\
\hline Yes & No \\
\hline$\downarrow$ & $\begin{array}{c}\downarrow \\
\text { Repair software and go to beginning of the } \\
\text { step } 3\end{array}$ \\
\hline \multicolumn{2}{|c|}{$\begin{array}{l}\text { Reduce field of scanning and scan short tracks in a different direction (axis). } \\
\text { All tracks are straight? }\end{array}$} \\
\hline \multirow[t]{2}{*}{ Yes } & $\begin{array}{c}\text { No } \\
\downarrow\end{array}$ \\
\hline & $\begin{array}{l}\text { Check precise synchronization of mirror } \\
\text { motion and vibrations using test patterns }\end{array}$ \\
\hline
\end{tabular}

\section{Test patterns for verification of scanning system, laser beam, and optical components}

To verify the optical system performance, different scan patterns on a test plate can be used (Fig. 6). Under prescribed spot size, laser power, and scanning speed, the laser beam should be tested on a familiar material with results known under optimal system performance to establish a meaningful baseline measurement.
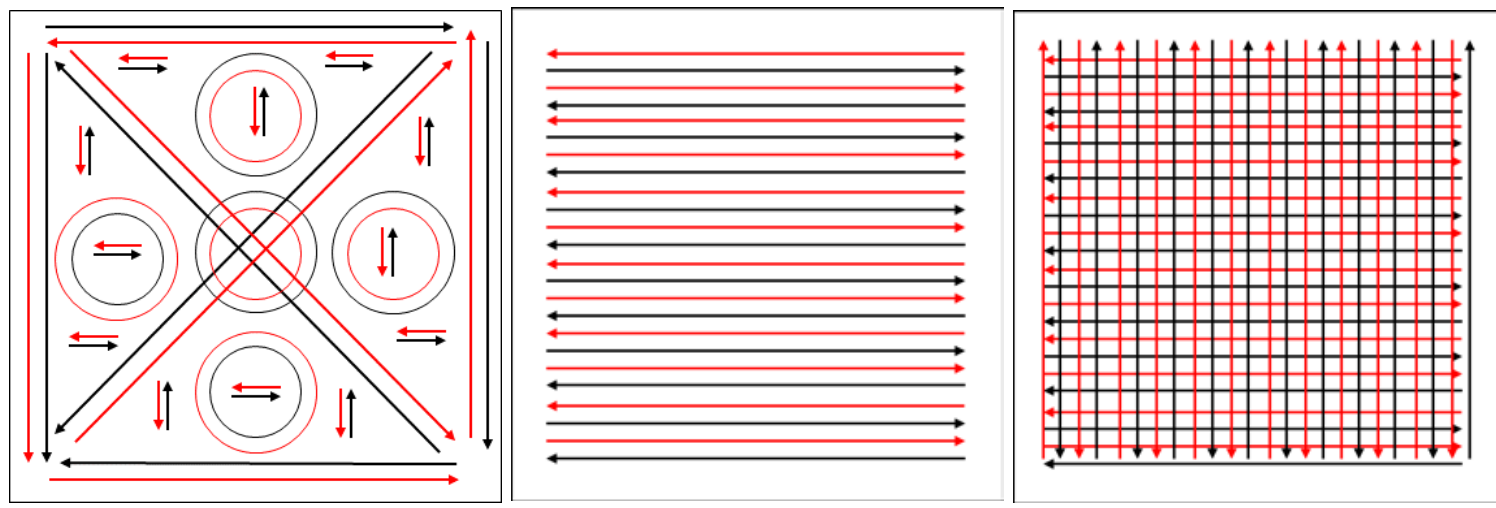

Fig. 6. Proposed test plate designs for verification of scanning system, laser beam, and optical components. Direction of scanning indicated by arrows, black lines are scanning at lower scanning speed and laser power, red lines are scanning at higher scanning speed and laser power.

Surface roughness of the substrate should be controlled and consistent with all diagnostic tests. It should be noted, that with powder experiments, higher absorption of the powder in comparison with solid material leads to higher temperatures in the process. Small nanoparticles of material that look like "smoke" and spatter formation cause polluted protective windows. Therefore, careful preparation of the system for experiments and protective atmosphere flows (air knife) are necessary. Chemical 
composition of the substrate and powder will influence the laser processing and resulting tracks. Sand-blasted substrates can result in embedded blast media material that can also change properties of melt pool, substrate materials, and eventual results of the singlelayer experiments.

\section{Conclusion}

Experiments with different materials, both on experimental and commercial systems, showed that stable operation of optical components is primarily important for producing defect-free LPBF objects with high repeatability and quality. The proposed workflow and patterns can be used to diagnose problems related to optical components in LPBF processes.

\section{References}

[1] Grantham S, Lane B, Neira J, Mekhontsev S, Vlasea M, Hanssen L (2016) Optical design and Initial Results from The National Institute of Standards and Technology's AMMT/TEMPS Facility. Proceedings of SPIE--the International Society for Optical Engineering 9738. https://doi.org/10.1117/12.2214246

[2] EOSINT M 280 Available at https://webbuilder5.asiannet.com/ftp/2684/TD_M280_en_2011-03-29.pdf

[3] International Organization for Standardization (2000) ISO/DIS 13694, Test method for laser beam parameters: Power (energy) density distribution (Geneva).

[4] International Organization for Standardization (1999) ISO/FDIS 11146, Lasers and laser-related equipment-Test methods for laser beam parameters-Beam widths, divergence angle and beam propagation factor (Geneva).

[5] Dini C (2018) Why Beam Analysis is Crucial for Additive Manufacturing. Laser Technik Journal 15(1):35-37. https://doi.org/10.1002/latj.201800004

[6] Zhirnov I V., Podrabinnik PA, Okunkova AA, Gusarov A V. (2015) Laser beam profiling: experimental study of its influence on single-track formation by selective laser melting. Mechanics \& Industry. https://doi.org/10.1051/meca/2015082

[7] Yadroitsev I, Yadroitsava I, Bertrand P, Smurov I (2012) Factor analysis of selective laser melting process parameters and geometrical characteristics of synthesized single tracks. Rapid Prototyping Journal.

https://doi.org/10.1108/13552541211218117

[8] Makoana N, Yadroitsava I, Möller H, Yadroitsev I (2018) Characterization of 174PH Single Tracks Produced at Different Parametric Conditions towards Increased Productivity of LPBF Systems-The Effect of Laser Power and Spot Size Upscaling. Metals. https://doi.org/10.3390/met8070475

[9] Shi W, Liu Y, Shi X, Hou Y, Wang P, Song G (2018) Beam diameter dependence of performance in thick-layer and high-power selective laser melting of Ti-6Al4V. Materials. https://doi.org/10.3390/ma11071237 
[10] Faidel D, Laskin A, Behr W, Natour G (2016) Improvement of selective laser melting by beam shaping and minimized thermally induced effects in optical systems. International Conference on Photonic Technologies LANE (Bayerisches Laserzentrum GmbH). Available at https://www.laneconference.org/app/download/11537218949/LANE2016_11258_Faidel_IC_endfor mat.pdf?t=1490712800

[11] Hansen KR, Alkeskjold TT, Broeng J, Lægsgaard J (2011) Thermo-optical effects in high-power ytterbium-doped fiber amplifiers. Optics express. https://doi.org/10.1364/OE.19.023965

[12] Bonhoff T, Schniedenharn M, Stollenwerk J, Loosen P (2017) Experimental and theoretical analysis of thermo-optical effects in protective window for selective laser melting. Proceedings of the International Conference on Lasers in Manufacturing LiM (Munich), pp 26-29. Available at https://www.wlt.de/lim/Proceedings2017/Data/PDF/Contribution31_final.pdf

[13] Yeung H, Neira J, Lane B, Fox J, Lopez F (2016) Laser Path Planning and Power Control Strategies for Powder. Proceedings of the Solid Freeform Fabrication Symposium

[14] ScanLab (2007) Installation and Operation Single Axis Module analog, YAG, 10 mm, with miniSSV amplifier board. Available at www.scanlab.deinfo@scanlab.de

[15] Koglbauer A, Wolf S, Märten O, Kramer R (2018) Investigation on laser scanner synchronization via advanced beam path analysis in 3D additive manufacturing systems. Journal of Laser Micro Nanoengineering. https://doi.org/10.2961/jlmn.2018.02.0015

[16] Engelmayer A (2005) New development and applications in scan head technology. Proceedings of ALAC 2005, pp 1-7. 